\title{
Research progress on factors affecting oxygen corrosion and countermeasures in oilfield development
}

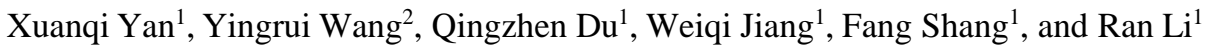 \\ ${ }^{1}$ Engineering Technology Research Institute of Huabei Oilfield Company, Renqiu 062552, China; ${ }^{2}$ Cooperative development project \\ department of Huabei Oilfield Company, Renqiu 062552, China
}

\begin{abstract}
Water injection, air injection, air foam injection, and in-situ combustion technology are used step by step in the later period of oilfield. Oxygen corrosion caused by different development methods has become a problem that could not be ignored. In this paper, the mechanism of oxygen corrosion, the influencing factors of oxygen corrosion and the new progress of oxygen corrosion countermeasures in recent years are systematically analyzed. Anticorrosion methods for different development modes are put forward. The direction of further research on oxygen corrosion in oilfield development is proposed: $\$$ Prediction and control of corrosion in oxygen environment need to be further studied to better guide corrosion protection in high temperature injection and production of oil and gas wells. 2 The research of anticorrosive coating materials and corrosion inhibitor technology needs to be further developed in the direction of safety and environment-friendly.
\end{abstract}

\section{Introduction}

Water injection, air injection, air foam injection, and in-situ combustion technology are used step by step in the later period of oilfield. With the development methods constantly updated, the recovery efficiency is obviously promoted. But oxygen corrosion caused by different development methods has become a problem that could not be ignored. In the process of water injection, the change of dissolved oxygen content in water quality will cause serious corrosion. In the process of air injection, air foam injection and in-situ combustion, high temperature and high pressure oxygen corrosion environment will be formed in oil and gas wells. However, experts and scholars at home and abroad mostly study the corrosion of $\mathrm{H} 2 \mathrm{~S} / \mathrm{CO} 2$ in the process of oilfield development, few studies have been done on the influencing factors of oxygen corrosion and its control measures. Therefore, it is necessary to study the influencing factors of oxygen corrosion in the process of oilfield development and put forward corresponding protective measures.

\section{Oxygen corrosion mechanism}

Oxygen corrosion refers to oxygen entering into the solution through the air/solution interface to the metal surface during the transportation of oxygen ${ }^{[1]}$, then migrating through the main layer of corrosive medium to the diffusion layer by convection, finally, the negative reduction reaction occurring on the metal surface during the diffusion to the metal surface. In neutral and alkaline solutions, oxygen can directly oxidize the $\mathrm{Fe}^{2+}$ produced by the anodic reaction to $\mathrm{Fe}^{3+}$. The cathodic reaction product $\mathrm{OH}^{-}$reacts with $\mathrm{Fe}^{3+}$ to form $\mathrm{Fe}(\mathrm{OH})_{3}$ precipitation, partial $\mathrm{Fe}(\mathrm{OH})_{3}$ precipitation is formed by direct hydrolysis of $\mathrm{Fe}^{3+}$. After that, some of the $\mathrm{Fe}(\mathrm{OH})_{3}$ precipitation produce $\mathrm{Fe}_{2} \mathrm{O}_{3}$ and $\mathrm{FeO}(\mathrm{OH})$ by hydrolysis or dehydration. At the same time, $\mathrm{FeO}(\mathrm{OH})$ can react with $\mathrm{Fe}^{2+}$ to form black and compact $\mathrm{Fe}_{3} \mathrm{O}_{4}$, which can hinder the penetration of eroded ions. But in the products, $\mathrm{Fe}_{2} \mathrm{O}_{3}$ and $\mathrm{FeO}(\mathrm{OH})$ are porous materials, which are easy to be penetrated by corrosive media and have poor adhesion to the matrix, so the corrosion products could not inhibit the further corrosion of pipelines. The specific reaction formula is as follows:

Anodic reaction: $\mathrm{Fe} \rightarrow \mathrm{Fe}^{2+}+2 \mathrm{e}^{-}$

Cathodic reaction:

$\mathrm{O}_{2}+2 \mathrm{H}_{2} \mathrm{O}+4 \mathrm{e}^{-} \rightarrow 4 \mathrm{OH}^{-}$

$4 \mathrm{Fe}(\mathrm{OH})_{2}+\mathrm{O}_{2}+2 \mathrm{H}_{2} \mathrm{O} \rightarrow 4 \mathrm{Fe}(\mathrm{OH})_{3}$

$2 \mathrm{Fe}(\mathrm{OH})_{3} \rightarrow \mathrm{Fe}_{2} \mathrm{O}_{3} \cdot \mathrm{H}_{2} \mathrm{O}+2 \mathrm{H}_{2} \mathrm{O}$

$\mathrm{Fe}(\mathrm{OH})_{3} \rightarrow \mathrm{FeO}(\mathrm{OH})+\mathrm{H}_{2} \mathrm{O}$

$8 \mathrm{FeO}(\mathrm{OH})+\mathrm{Fe}^{2+}+2 \mathrm{e}^{-} \rightarrow \mathrm{Fe}_{3} \mathrm{O}_{4}+4 \mathrm{H}_{2} \mathrm{O}$

In acidic solution, the first intermediate product of $\mathrm{O}_{2}$ in cathodic reduction reaction is $\mathrm{HO}_{2}{ }^{-[2]}$. After that, $\mathrm{HO}_{2}{ }^{-}$ is acidified to $\mathrm{H}_{2} \mathrm{O}_{2}$, and $\mathrm{H}_{2} \mathrm{O}_{2}$ is further reduced to $\mathrm{H}_{2} \mathrm{O}$ by $\mathrm{H}^{+}$. The whole reaction process is a corrosion process of continuous acidification and reduction. The specific reaction formula is as follows:

Anodic reaction: $\mathrm{Fe} \rightarrow \mathrm{Fe}^{2+}+2 \mathrm{e}^{-}$

Cathodic reaction:

$\mathrm{O}_{2}+\mathrm{e} \rightarrow \mathrm{O}_{2}^{-}$

$\mathrm{O}_{2}{ }^{-}+\mathrm{H}^{+} \rightarrow \mathrm{HO}_{2}$

$\mathrm{HO}_{2}+\mathrm{e} \rightarrow \mathrm{HO}_{2}$ 


$$
\begin{aligned}
& \mathrm{HO}_{2}{ }^{-}+\mathrm{H}^{+} \rightarrow \mathrm{H}_{2} \mathrm{O}_{2} \\
& \mathrm{H}_{2} \mathrm{O}_{2}+2 \mathrm{H}^{+}+2 \mathrm{e} \rightarrow 2 \mathrm{H}_{2} \mathrm{O}
\end{aligned}
$$

\section{Influencing factors of oxygen corrosion}

\subsection{Dissolved oxygen content}

The solubility of oxygen in solution and the partial pressure of oxygen on the surface of solution adheres to Henry's law, that is, the dissolved oxygen content in corrosive solution is proportional to the partial pressure of oxygen on the surface of solution. Owing to the cathodic reduction reaction of $\mathrm{O}_{2}$ in corrosive solution, electrons in cathodic region are continuously consumed, which leads to the dissolution of electrons in the anodic region and the loss of electrons. Therefore, even a small amount of dissolved oxygen will cause very serious corrosion to the tubing of oil and gas wells. It is found that the corrosion rate increases rapidly with the increase of dissolved oxygen content in different corrosion environments, and there is a positive correlation between the corrosion rate and dissolved oxygen content ${ }^{[3-5]}$. When the dissolved oxygen content reaches a certain value, a corrosion product film is formed on the surface of steel, which inhibits metal corrosion ${ }^{[6]}$ and slows down the corrosion rate; when the dissolved oxygen content continues to increase, the corrosion medium penetrates the product film due to the film's unstability, so the dissolved oxygen content will accelerate the metal corrosion $^{[7-10]}$. Zhouquan ${ }^{[11]}$ found that when the dissolved oxygen content is less than $0.1 \mathrm{mg} / \mathrm{L}$, the corrosion rate of matals is smaller, when the dissolved oxygen content reaches $860 \mathrm{mg} / \mathrm{L}$, the corrosion of metals is yet inhibited, when the dissolved oxygen content exceeds $10100 \mathrm{mg} / \mathrm{L}$, the corrosion of metals is accelerated.

\section{$3.2 \mathrm{pH}$ value}

In acidic solution with $\mathrm{pH}$ less than 4 , the cathodic reaction is hydrogen evolution reaction, which is mainly uniform acid corrosion. In this solution, the corrosion rate increases sharply with the decrease of $\mathrm{pH}$ value. When the $\mathrm{pH}$ is between 4 and 10, the concentration of hydrogen ion decreases, and the cathodic reaction is dominated by oxygen uptake reaction. The corrosion is controlled by the diffusion of oxygen, and the surface is formed by hydrated iron oxide film. Therefore, the corrosion rate remains constant regardless of $\mathrm{pH}^{[12]}$. In this situation, corrosion is not affected by the type and structure of steel, but only affected by dissolved oxygen, temperature and flow rate. Then, with the increase of $\mathrm{pH}$ value, the $\mathrm{OH}^{-}$concentration increases, when the $\mathrm{pH}$ value is in the alkaline range of 10-13, a dense oxide film $\gamma-\mathrm{Fe}_{2} \mathrm{O}_{3} / \mathrm{Fe}_{3} \mathrm{O}_{4}$ can be formed on the metal surface, and the corrosion rate decreases ${ }^{[13,14]}$. If the $\mathrm{pH}$ continues to rise, the corrosion rate will increase again due to the following reactions:

$\mathrm{Fe}_{3} \mathrm{O}_{4}$ (main component of the dense oxide film)
$+4 \mathrm{NaOH} \rightarrow 4 \mathrm{NaFeO}_{2}+\mathrm{Na}_{2} \mathrm{FeO}_{2}+2 \mathrm{H}_{2} \mathrm{O}$

\subsection{Temperature}

In a closed system, when the oxygen content is constant, the dissolved oxygen content will not change with the increase of temperature. But with the increase of temperature, the activity of oxygen molecules increases, and the diffusion coefficient of oxygen increases, too. The acceleration of depolarization reaction leads to the increase of corrosion rate. There is a linear relationship between temperature and corrosion rate ${ }^{[15,16]}$, and the corrosion products are relatively soft. When the temperature rises to a certain value, the corrosion products are more hard and dense, mainly $\mathrm{Fe}_{3} \mathrm{O}_{4}$, which hinders the further occurrence of corrosion, so the corrosion rate will not increase significantly ${ }^{[17]}$. In the open system, when the $\mathrm{pH}$ value is $4-10$, the dissolved oxygen content decreases with the increase of temperature, but at the same time the reaction rate of electrochemical corrosion accelerates. The overall trend is that the corrosion rate of carbon steel is increasing with the increase of temperature, reaching the maximum at $80^{\circ} \mathrm{C}$. But when the temperature is above $80^{\circ} \mathrm{C}$, the corrosion rate decreases sharply due to the sharp decrease of dissolved oxygen content ${ }^{[18]}$.

\subsection{Salinity}

There are many kinds of ions in formation water of oilfield, among which the most important factor affecting corrosion rate is $\mathrm{Cl}^{-}$and $\mathrm{SO}_{4}{ }^{2-}$. One theory is that the electronegativity of $\mathrm{Cl}^{-}$and $\mathrm{SO}_{4}{ }^{2-}$ is very strong, and their adsorption capacity is much greater than that of dissolved oxygen on metal surface, which enhances the conductivity of solution ${ }^{[19,20]}$. Therefore, $\mathrm{Cl}^{-}$and $\mathrm{SO}_{4}{ }^{2-}$ will hinder the formation of passive film, resulting in the increase of corrosion. The other theory holds that $\mathrm{Cl}^{-}$ radius is very small, and it is easy to penetrate the passive film, which intensifies the competition between the dissolution and formation of the film. When $\mathrm{Cl}^{-}$ concentration reaches $0.2 \mathrm{~mol} / \mathrm{L}$, the dissolution rate of $\mathrm{Fe}(\mathrm{OH})_{2}$ film is equal to its formation rate, and the passive film could not be formed at all. The passivation phenomenon disappears ${ }^{[21]}$, which leads to the increase of corrosion rate.

\subsection{Current velocity}

With the increase of current velocity, the diffusion of oxygen and reaction speed raises. When the medium movement transits from laminar to turbulent flow, the turbulent kinetic energy increases with the enhancement of turbulent motion, which enhances the shear stress on the surface of carbon steel, increases the amount of oxygen transferred to the surface of carbon steel, accelerates the dissolution of ferrite near cementite ${ }^{[22,23]}$, and leads to the intensification of corrosion reaction. However, when the current velocity increases to a certain critical value and the concentration of dissolved oxygen on the metal surface reaches the critical concentration 
which can passivate the metal, the formation of passive film on the metal surface is promoted and the corrosion is slowed down. As the flow rate continues to increase, the passive film is destroyed by erosion, and the corrosion rate rises again ${ }^{[24]}$.

\section{Research progress of oxygen corrosion control}

\subsection{Corrosion-resistant steel}

At present, corrosion-resistant steels used for oxygen corrosion control are mainly Cr-containing alloys, such as $90 \mathrm{H}-9 \mathrm{Cr}, 90 \mathrm{H}-13 \mathrm{Cr}, 1 \mathrm{Cr} 18 \mathrm{Ni} 9 \mathrm{Ti}, 304,316$. The results show that the uniform corrosion rate of the above five steel sheets under different conditions of in-situ combustion or air injection is all less than $0.2 \mathrm{~mm} / \mathrm{a}$, and they all have good corrosion resistance $[6,25,26]$. D. Huenert et al. ${ }^{[27]}$ carried out corrosion experiments under different oxygen partial pressures in dynamic reactor by using steel sheets with different $\mathrm{Cr}$ content. It was found that the higher the $\mathrm{Cr}$ content of steel sheets, the smaller the effect of oxygen on their corrosion. Cr-containing alloys can effectively inhibit oxygen corrosion. The reason is that $\mathrm{Cr}$ is easy to accumulate in the corrosion product film and form hydroxides or oxides of $\mathrm{Cr}$. These compounds can form a dense protective layer, which can prevent the transport process between ions in solution and metal surface, thus playing a good role in inhibiting corrosion [28]. Although the corrosion resistance of Cr-containing alloys is very strong, its shortcomings are high cost, poor economy, and low production wells are not suitable for application.

\subsection{Coating technology}

The coatings for oxygen corrosion can be divided into two categories: non-metallic coatings and metal coatings. Non-metallic coatings mainly include silicate coatings, epoxy resin coatings and polyethylene anticorrosive coatings ${ }^{[29]}$. Metal coatings mainly include tungsten alloy, nickel-phosphorus alloy, nickel-tungsten alloy, nickel-tungsten-phosphorus alloy and so on, which can be realized by electroplating. HAN Xia ${ }^{[26]}$ simulated the field test conditions of combustion-drive in laboratory, and screened and evaluated different anticorrosion coatings and coatings. It was found that epoxy powder coatings and nickel-tungsten alloy coatings had good protection to $\mathrm{N} 80$ and $\mathrm{P} 110$ in high pressure oxygen, tap water and $60{ }^{\circ} \mathrm{C}$ corrosion environment, while the protection of nitriding and nickel-phosphorus alloy coatings was poor. In order to solve the problem of oxygen corrosion in gas injection wells during air flooding, FENG Zhaoyang et al. ${ }^{[30]}$ proposed that the use of coating tubing and the installation of filters at the wellhead could avoid the corrosion product iron oxide blocking the pipeline and protect the gas injection pipeline. But at present, non-metallic coatings and metal coatings are seldom used in engineering cases. The main reason is that in the process of transportation and operation of oil and casing tubes, it is easy to cause local peeling of coatings and pitting corrosion.

\subsection{Cathodic protection}

The main principle of cathodic protection is to convert the whole metal surface into cathode, that is, to pass enough applied current to the protected equipment. So, there is no electric current flowing out of the metal surface. There are two ways of cathodic protection, sacrificial anode protection and forced current protection. The difference between the two ways is that the source of cathodic protection current is different. The cathodic protection current of sacrificial anode method comes from sacrificial anodes such as zinc, magnesium and aluminium, and the cathodic protection current of forced current method comes from DC power. Oil and gas pipelines are usually protected by forced current as the main method and sacrificial anode as the auxiliary method ${ }^{[33]}$. On the basic of lab research, GENG Shijiang et al. ${ }^{[34]}$ selected an oxygen corrosion resistant sacrificial anode material for air foam flooding system. The material protection can reach $98 \%$ at $95^{\circ} \mathrm{C}$, and its dissolution is uniform, and the electrochemical output performance is well. However, sacrificial anode is not suitable for high resistance environment, the protection current of the whole cathodic protection system could not be adjusted, and the quality of the pipeline itself is required to be good enough.

\subsection{Addition of corrosion inhibitor}

Adding corrosion inhibitor can form a passivation film in the surface of carbon steel, which can slow down the corrosion. At present, the main way to protect oil, gas and water wells string is to add corrosion inhibitor in the annular space between the tubing and casing.

WANG $\mathrm{Na}$ et al. ${ }^{\left[{ }^{35]}\right.}$ designed and synthesized a new type of corrosion inhibitor according to the corrosion environment characteristics of high salinity, high $\mathrm{Cl}^{-}$, low $\mathrm{pH}$ value and excessive dissolved oxygen content in water injection system of Tahe oilfield. The corrosion inhibition performance was improved by changing to hydrophilic substituent groups on imidazoline ring. When the concentration reached $100 \mathrm{mg} / \mathrm{L}$, the corrosion rate was reduced to below $0.076 \mathrm{~mm} / \mathrm{a}$ and the corrosion inhibition rate could reach over $88 \%$. To solve the problem of serious equipment corrosion caused by high oxygen content in seawater during oil displacement, LI Yong et al. ${ }^{[36]}$ developed a HC-3 deoxidizer which is composed of sodium sulfite, sodium bisulfite and acetone oxime. When the dosage was $70 \mathrm{mg} / \mathrm{L}$, the seawater with $8 \mathrm{mg} / \mathrm{L}$ oxygen content could be deoxidized to $0.04 \mathrm{mg} / \mathrm{L}$, and deoxidization rate could reach $99.5 \%$.

LI Ying et al. ${ }^{[37]}$ developed four corrosion inhibitors to solve the problem of oxygen corrosion in production wells during in-combustion process. The inhibitors were screened under the conditions of temperature $160^{\circ} \mathrm{C}, \mathrm{CO}_{2}$ partial pressure $0.1 \mathrm{MPa}$ and $\mathrm{O}_{2}$ content $0.1 \%$. The experimental results show that the corrosion inhibition rate of QH-01could reach over $90 \%$. 
LIN Weimin et al. ${ }^{[38]}$ developed a DH-1 inhibitor to solve the problem of oxygen corrosion in the process of air foam injection. Field tests showed that in the air injection environment, owing to high temperature of the injection system and fast flow rate of the corrosion medium, the inhibitor could not form a protective coating on the surface of the steel. Although the corrosion rate decreased after adding the inhibitor, the inhibition rate was still less than $70 \%$, which could not meet the site requirement. Thus, it can be seen that in the process of air foam injection, adding inhibitors as a single method could not achieve the purpose of anticorrosion, and it needs to cooperate with other anticorrosive method. Although the cost of corrosion inhibitors is not high in the early stage, with the increase of oil field exploitation, investment cost will be higher and higher. And the versatility is poor, different corrosion inhibitors need to be prepared for different water types. Adding corrosion inhibitors also may cause pollution of stratum and natural environment.

\subsection{Polyethylene lined tubing and FRP tubing}

Both polyethylene lined tubing and FRP tubing have the advantages of strong corrosion resistance, good thermal insulation, smooth inner wall and low fluid resistance. However, owing to complex shrinkage processing technology, poor high temperature resistance of polyethylene lined tubing and high cost, low tensile strength of FRP tubing ${ }^{[39]}$, polyethylene lined tubing and FRP tubing had less application in oilfield production and were mainly used in water wells.

\subsection{Compressor dewatering process}

It is found that when the injected air was separated by multi-stage cooling by compressor to ensure that the injected air was dry air, and no serious corrosion occurred after injecting the dry air into the wellbore ${ }^{[40]}$. HUANG Jihong et al. ${ }^{[41]}$ used air compressor to dry the injected air in the combustion-drive test, and maintained the injected air in a relatively low pressure, low temperature and dry state, excluding the formation of dissolved oxygen. It was found that there was no obvious corrosion in the gas injection wells. Researchers of China University of Petroleum simulated the field environment of air flooding in oil field, carried out corrosion experiments with $\mathrm{N} 80, \mathrm{P} 110$ and $3 \mathrm{CrN} 80$ materials at $120^{\circ} \mathrm{C}, 35 \mathrm{MPa}$ and $3-10 \%$ oxygen content(drying of the reactor was done before the experiment to ensure that there was no water vapor in the test process). The experimental results are shown in Figure 1.

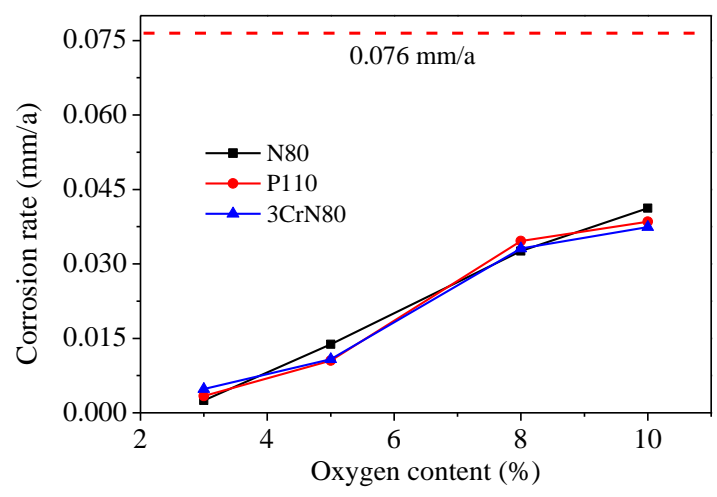

Fig.1. Oxygen corrosion rate curves of different materials in dry environment

It can be seen that the corrosion rate of the three materials increases gradually with the increase of oxygen content, and there is no obvious difference in the corrosion rate. The corrosion rate of the materials with $10 \%$ oxygen content is about $0.04 \mathrm{~mm} / \mathrm{a}$. It shows that the three materials do not suffer from oxygen corrosion in completely dry environment, and no anticorrosion measures need to be taken.

\section{Conclusion}

In this paper, six kinds of anti-oxygen corrosion processes are analyzed. Each process has its applicability and limitations. Therefore, different anticorrosion methods can be selected according to the causes of oxygen corrosion in different development methods. In the process of water injection development, corrosion resistance pipes, corrosion inhibitor, cathodic protection, polyethylene tubing and other single methods can be used to slow down corrosion. In view of the complex corrosive environment such as air injection, air foam injection and in-situ combustion, the method of compressor dehydration should first be considered. Secondly, corrosion protection can be achieved by synergistic use of corrosion resistant pipes, adding corrosion inhibitors, and cathodic protection. Prediction and control of oxygen corrosion still need to be further studied to better guide corrosion protection in high temperature injection and production of oil and gas wells. Research on anticorrosive coating materials and corrosion inhibitor technology should be further developed in the direction of safety and environment-friendly.

\section{References}

1. Lu Z. Research on technology of hot dipped Galfan coating of sucker rods and its properties (China University of Petroleum, 2016)

2. Dun Z. Jiajia W. Cathodic dissolved oxygen reduction reaction in the corrosion process of marine environment (2016)

3. Azar, J.J. PEI, 3 (1979)

4. Chaoneng Z. ESR, 12, 54-55 (1999) 
5. Guangzhi L, Huaijun Y, Youwei J, et al. PED, 45, 105-110 (2018)

6. Haikui L. Prevention techniques of oxygen corrosion of down hole string in air injection process (China University of Petroleum, 2015)

7. MONTES A R, GUTIÉRREZ D, MOORE R G, et al. JCPT, 49, 56-63 (2010)

8. Brondel D, Edwards R, Hayman A. et al. OR, 4-18(1994)

9. B. Yu, D.Y. Li, A. Grondin. Wear. 302, 1609-1614 (2013)

10. Yuanyuan L, Chunmao Z, Suiping W, et al. CSPT, 20, 386-387 (2008)

11. Quan Z. Corrosion and anti-corrosion techniques for air injection EOR process (China University of Petroleum, 2009)

12. 《Drilling Manual (First party) 》writing group. Drilling Manual (First party) 460-518 (1990)

13. Chunlin L, Jianwen T, Zhifa G. OGSE, 19, 36-37(2000)

14. Qingwei S, Yun L, Xiuling C, et al. TCC, 22, 63-66(2008)

15. Xue L, Qingjie Z, Ning Z, et al. ST, 46, 210-212 (2017)

16. Weibing Z, Xuanzhou W, Weijian T, et al. CEOG, 47, 67-72 (2018)

17. Zenghua M, Yongtao S, Tao L, et al. PIA, 60-63 (2012)

18. Peng Z, Jie Y, Jinbao G. SP, 39, 29-33 (2010)

19. Zhen Z, Yuwu L. CSPT, 20, 260-264 (2008)

20. Lei W. CPM, 34, 1-4 (2006)

21. Zhaoyu D, Yunyu H, Huaixiang L. JSNU, 15, 274-278 (2000)

22. Yuzhen L, jingjun L, xingyue Y, et al. JCSCP, 19, 1-7 (1999)

23. xingyue Y, jingjun L, Yuzhen L, et al. JCSCP, 19, 8-14 (1999)

24. Jiangjiang Z. STR, 32, 65-70 (2014)

25. Wendong W, Qiang L, Liwen Z, et al. MME, 42, 11-16 (2018)

26. Xia H. CSPT, 22, 247-250 (2010)

27. Huenert D., Kranzmann A. CS, 53, 2306-2317 (2011)

28. Pfennig A, Bäßler R. CS, 51, 931-940 (2009)

29. Qingyu Z. Practical anti-corrosion technology for oil and gas field engineering (2009)

30. Zhaoyang F, Liping W, Gao L, et al. CR, 29, 62-66 (2015)

31. Zhaohui W, Huanqin Z, Baijian Y. PBT, 18, 36- 39 (2000)

32. Fengyun Q. ODGS, 10, 31- 32 (2001)

33. Zhiyuan $\mathrm{X}$, Wuxi B, Zhenhua $\mathrm{C}$, et al. OGST, 33, 938-944 (2014)

34. Shijiang G, Hua C. OGSE, 29, 79-80 (2010)
35. Na W, Zhiqiang L, Xing S, et al. CS, 27, 48-50 (2013)

36. Yong L, Mingliang Z. OC, 23, 140-144 (2006)

37. Ying L, Shulan L, Qiufang C. SOGR, 12, 101-103 (2005)

38. Weimin L, Xuefeng L, Xiuling C, et al. OC, 27, 342-345 (2010)

39. Yuanzhe Z, Xuesong L, Xiaoling R. PT, 21, 153-154 (2018)

40. Kui N, Shibao Y, Haiyan J. In-situ combustion technology \& practice (2010)

41. Jihong $\mathrm{H}$, Jinzhong $\mathrm{L}$, Lu L, et al. XJPG, 34, 101-104 (2013) 